\title{
CIRSI: the Cambridge infrared survey instrument for wide-field astronomy
}

Craig D. Mackay, Richard G. McMahon, Martin G. Beckett, Meghan Gray, Richard S. Ellis, et al.

Craig D. Mackay, Richard G. McMahon, Martin G. Beckett, Meghan Gray, Richard S. Ellis, Andrew E. Firth, M. Hoenig, James R. Lewis, Steve R. Medlen, Ian Robert Parry, Jim M. Pritchard, Chris S. Sabbey, "CIRSI: the Cambridge infrared survey instrument for wide-field astronomy," Proc. SPIE 4008, Optical and IR Telescope Instrumentation and Detectors, (16 August 2000); doi: $10.1117 / 12.395448$ 


\title{
CIRSI: the Cambridge Infrared Survey Instrument for Wide-Field Astronomy
}

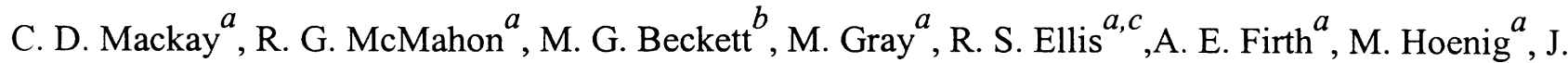 \\ R. Lewis ${ }^{a}$, S. R. Medlen ${ }^{a}$, I. R. Parry $^{a}$, J.M. Pritchard ${ }^{a}$ and C.S. Sabbey ${ }^{a}$. \\ ${ }^{a}$ Institute of Astronomy, University of Cambridge, Madingley Road, Cambridge CB3 0HA, UK. \\ Tel: + 44-1223 -337543, fax: + $44-1223-330804$ \\ $b$ \\ Carnegie Observatories, 813 Santa Barbara Street, Pasadena, California 91101, USA \\ ${ }^{c}$ California Institute of Technology, 1200 East California Boulevard, Pasadena, California 91125
}

\begin{abstract}
The search for galaxies at redshifts $>3$ becomes increasingly difficult in the visible since most of the light emitted by these objects is redshifted into the near infrared (wavelengths $>1$ micron). The recent development of high-performance near infrared arrays has made it practical to build a wide field survey instrument for operation in the near infrared part of the spectrum. CIRSI, the Cambridge Infrared Survey Instrument, uses four of the HAWAII-1 MCT arrays each of which has 1024 by 1024 pixels. This paper describes a number of the novel features of CIRSI and summarises the present performance achieved by CIRSI and the scientific programmes it is principally engaged in.
\end{abstract}

\section{SCIENTIFIC REQUIREMENTS FOR AN EXTRAGALACTIC INFRARED SURVEY INSTRUMENT}

An important field of astronomical research is the understanding of the way that the structure of galaxies and clusters of galaxies developed with time. Although the structure of galaxy clusters has been studied in great detail at lower redshifts, the discovery of galaxies with redshifts $>3$ has made it very important to study the structure of galaxy clusters at these redshifts. In 1996, the need to try to carry out wide field surveys in the J-band (1.3 microns) and H-band (1.6 microns) became a major scientific driver. At about the same time Rockwell Science Centre in Thousand Oaks, California had begun manufacturing high efficiency, low noise large area detectors that work over the wavelength range of $0.85-2.5$ microns. The sensitivity of an imaging system for detecting the high redshift galaxies that we are interested in is much poorer in the longer wavelength regions (the $\mathrm{K}$ band centred on 2.2 microns) because of the much higher thermal background encountered and so there was no scientific need for us to make observations at wavelengths longer than about 1.8 microns, the edge of the $\mathrm{H}$ band.

As with all survey instruments there is a complicated trade-off between using an image scale that maximises the field of view and the need to use pixels small enough to sample the telescope image properly so as to give the best possible sensitivity for faint objects in a given observing time. These considerations meant that the ideal telescope size for survey work such as this is in the 2.5-4 metre range. Telescopes that were most directly accessible to our group were the Isaac Newton Telescope (2.5 $\mathrm{m}$ ) and the William Herschel Telescope $(4.2 \mathrm{~m})$ on La Palma as well as the Du Pont Telescope (100 inch or $2.5 \mathrm{~m})$ at Las Campanas in Chile. The two telescopes on Le Palma had prime foci that would allow a wide field of view with a pixel size of 0.46 arc seconds, while the DuPont telescope at Cassegrain had a pixel size of 0.17 arc seconds. The need to work at prime focus on the La Palma telescopes made it additionally difficult to design and instrument that would operate in the $\mathrm{K}$ band since it would be necessary to provide a reimaging system that had a cold stop in order to minimise the thermal background in the camera. Given the scientific requirements listed above it was decided to design CIRSI only to work out to $\mathrm{H}$ band and not at longer wavelengths. 
A further requirement was that the instrument should be designed as a fast track project. One of the principal competitive edges that a University research group can have over the major national facilities is the speed with which they can get a novel instrument onto a telescope. This was made possible in this case by having a relatively specific range of scientific goals and being able to build an instrument to the less demanding standards that are appropriate for an instrument that does not need to be operated in a wide range of modes by observers with a wide range of technical skills.

\section{FOCAL PLANE ARRANGEMENT}

The Institute of Astronomy, University of Cambridge, was very fortunate to receive a substantial donation from the Raymond and Beverly Sackler foundation in support of the construction of this instrument. The level of funding available allowed us to use four of the HAWAII-1 infrared arrays each of which has 1024 by 1024 pixels of 18.5 microns. These devices are not capable of being butted as they have readout electrodes on all four sides, but they may be packet into a convenient format so that each sensitive area of 1024 by 1024 pixels is separated from the next by approximately 950 pixels. The general layout is shown in figure 1.

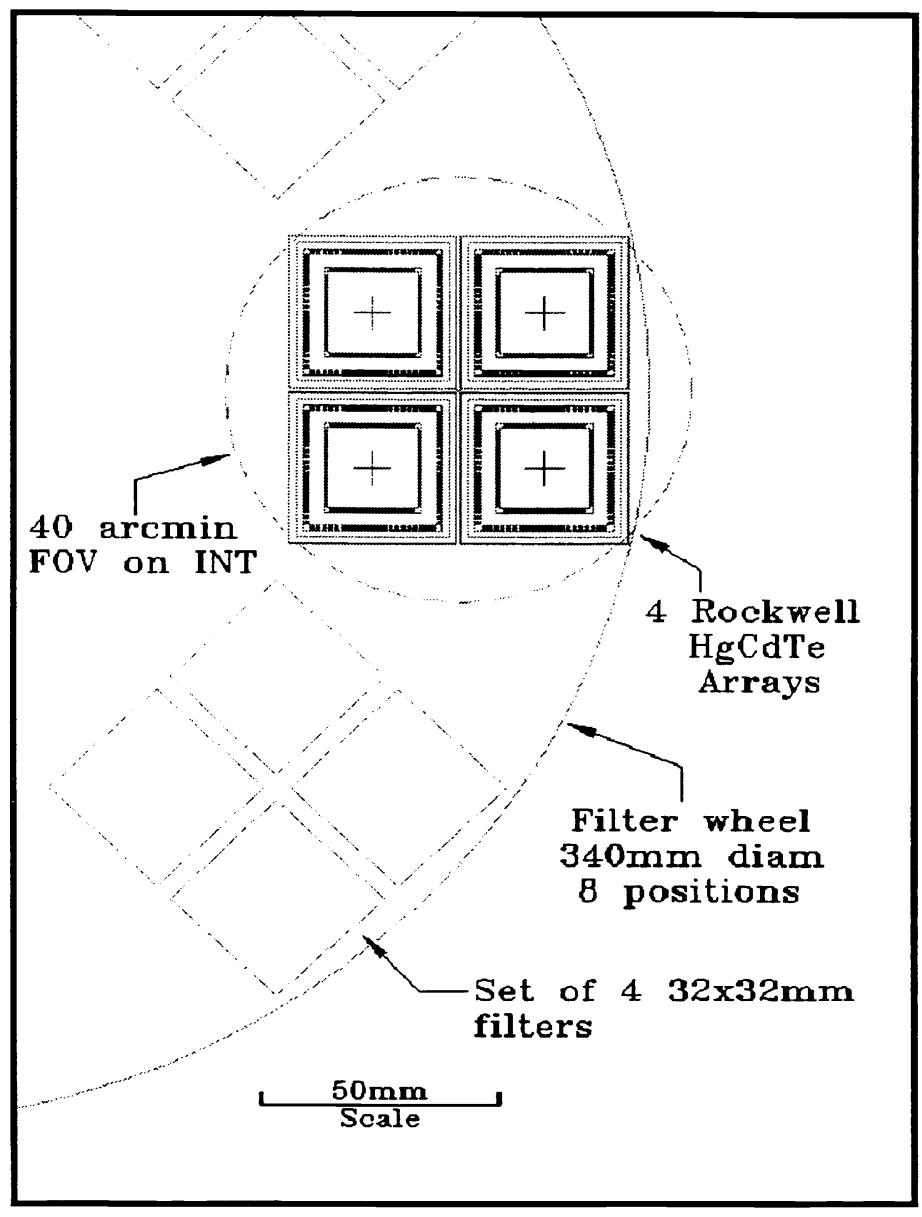

Figure 1. The general layout of the focal plane of CIRSI, viewed from above. The four detectors are packed as closely as possible, and the filter wheel has eight positions, each of which has four filters, one for each detector.

The HAWAII-1 arrays need to be operated at approximately liquid nitrogen temperature (-196 C) in order to give a dark current which is acceptably low. The layout of the four detectors let us use separate filters for each detector giving significant economies. The filters were mounted in a filter wheel with a total of 8 positions. One of these positions is a metal blanking plate which lets us measure the dark current of the detector array in the absence of any background signal (in $\mathrm{H}$ band the thermal radiation detected by these devices amounts to several hundred photons per pixel per second). No shutter is included in the system has these are CMOS devices that are X-Y addressable so that smearing does not occur during readout if light falls on the detectors as with CCDs. 
The back focal distance behind the prime focus correctors of both the Isaac Newton Telescope and the William Herschel Telescope is relatively short. The filter wheel and detector assembly are kept as close to liquid nitrogen temperatures as possible by carefully shielding the entire assembly, and background radiation levels from the telescope structure, etc., are minimise by using as much baffling close to the focal plane as practicable, given the back focal distance restrictions.

Significant problems were experienced in procuring filters which were good enough in terms of their blocking performance. The detectors have a small but significant sensitivity at wavelengths longer than 2.5 microns. Maximum transmission may be achieved if the filters are designed to have good blocking provided by a multilayer coating. However it is important to remember that rays that strike the filters from more acute angles than those that come from the telescope mirror directly will have their blocking edge shifted to shorter wavelengths than rays incident perpendicular to the filter. Blocking for these wide angle rays can be inadequate unless filters are properly specified and properly designed by the manufacture.

The vacuum dewar was designed with an aluminium outer body and a copper liquid nitrogen in cylinder. The liquid nitrogen cylinder is supported away from the outer body with a fibreglass spider. The assembly which holds the detectors and the filter wheel assembly is supported from the front of the outer body by a series of cantilevered epoxy graphite struts. This gives extremely good stability in all axes for the position of the focal plane of the instrument. In order to allow operation of the dewar in an inverted position or in a sideways looking configuration, with the liquid nitrogen fill pipes below the front of the dewar, the liquid nitrogen cylinder was constructed with copper fins which extended from the cold surface of the cylinder almost the full length of the cylinder back towards the fill pipes. This ensured that as good a thermal contact between the cold surface and the liquid nitrogen remaining in the dewar, in inverted position, was enough to maintain a working temperature that was essentially independent of orientation. CIRSI gives a whole time on one fill of liquid nitrogen in excess of 30 hours in downward looking mode and approximately 24 hours in upward looking mode.

\section{DETECTOR CONTROLLER ARRANGEMENTS}

Each of the four 1024 by 1024 detectors has four essentially independent quadrants of 512 by 512 pixels. Our scientific programmes meant that the fastest readout rates were not essential for CIRSI. In order to minimise development time we decided to use a commercial CCD controller manufactured by PerkinElmer Life Sciences, formerly AstroCam Ltd., in Cambridge, England. The Capella 4100 controller has a single signal input capable of digitising with 14 bit accuracy at up to an $8 \mathrm{MHz}$ pixel rate. We constructed an interface box which multiplexed each of the 16 quadrants sequentially onto a common analogue line to feed the controller as well as providing the correct logic and bias levels for the detectors. The interface box also provided reference analogue signal levels with a dummy output circuitry designed to mimic the output from the HAWAII-1 detectors (this capability is now provided as part of the HAWAII-2 1024 by 1024 detector multiplexers). The system is designed with no electronics of any sort inside the vacuum dewar. The output impedance of the on chip buffer amplifier is low enough to make it unnecessary to have any buffer electronics contained within the dewar.

In order to minimise the possibility of any glow being observed from any of the detectors, each output transistor is only connected to the readout electronics when that quadrant is being readout. At all other times it is isolated. Miniature reed relays were used for this purpose because they give extremely high isolation. In addition the supply voltages that provide of the analog and digital bias supplies were made to be variable. Although it is usual to operate digital electronics at $5 \mathrm{~V}$, reducing the voltage level significantly can greatly reduce the glow from the shift registers on the edge of the device. This glow can be at least as important as the glow from the output transistors and the ability to adjust the drive voltage was extremely helpful. If this drive voltage is reduced too far then of course clocking no longer happens properly at provided it is set at a level significantly above this threshold then there is no reason not to use it. In practice we found that operating the detectors at closer to $4 \mathrm{~V}$ than $5 \mathrm{~V}$ made a marked difference to the amount of shift register glow we experienced. The microcode which runs in the sequencer in the Capella controller was substantially rewritten in order to handle the rather different readout requirements of these infrared detectors. The Capella 4100 is controlled by an interface card and the data returned to the host computer via a PCI interface board within the host PC computer.

The system allowed pixel readout rates of between $118 \mathrm{kHz}$ and $1.7 \mathrm{MHz}$ although the performance of the detectors required that most of the observations were done at the lower end of this range in order to minimise the smearing which was seen in some cases close to bright (hot) pixels. In addition, the readout noise of the system is significantly better at lower pixel rates (Beckett et al., 1998) ${ }^{1}$. Typical readout times are approximately 12 seconds for all four detectors. 
The raw data from these devices suffers from a very substantial fixed pattern noise principally caused by a combination of pixel to pixel nonuniformities and fixed differences in the reset levels achieved on each pixel. The output data are derived by subtracting an image from an earlier one taken immediately after the array has been reset. CIRSI is run in two principal modes. The simplest involves resetting the arrays and then reading them sequentially several times at fixed intervals until the end of the exposure. This nondestructive read gives a sequence of images where the signal in each pixel progressively increases from image to image in the sequence. In cases where the sky background is bright or where brighter objects are being observed a different readout strategy may be used. In this each quadrant is read in the following way. Each row of the quadrant is read, that row is reset, and then read again. This is repeated for each row in the quadrant giving a dataset consisting of 512 rows each of which is 1024 pixels wide. The reset corrected images are obtained from this read-reset-read detector readout mode by subtracting the first 512 pixels of each row from the second 512 pixels of the previously read out image of the same quadrant. This method has the advantage of giving the widest dynamic range and ensured wasting virtually no telescope time between resetting a row and reading the values to which that row had been reset.

We have found some problems in operating the HAWAII-1 devices as follows. If the device has not been operated for even a short period of time then when it starts being clocked there is a significant transient in the output bias level which takes a fraction of the second to settle to a stable value. Any differences in clocking means that the reset subtraction essential to getting good reset subtraction will be slightly less good if the read and reset images were not taken under conditions of identical clocking and identical clocking history. In this respect it is important to remember that the recent clocking history of the device is critical. The consequence of this is that we find that the read-reset-read method gives slightly more sensitivity to the start-up output bias transient than the more straightforward nondestructive read method. There is no problem in coping with these transient provided one is aware that they are present. The transient varies relatively slowly and therefore is easy to model and subtract out as part of the standard data processing pipeline.

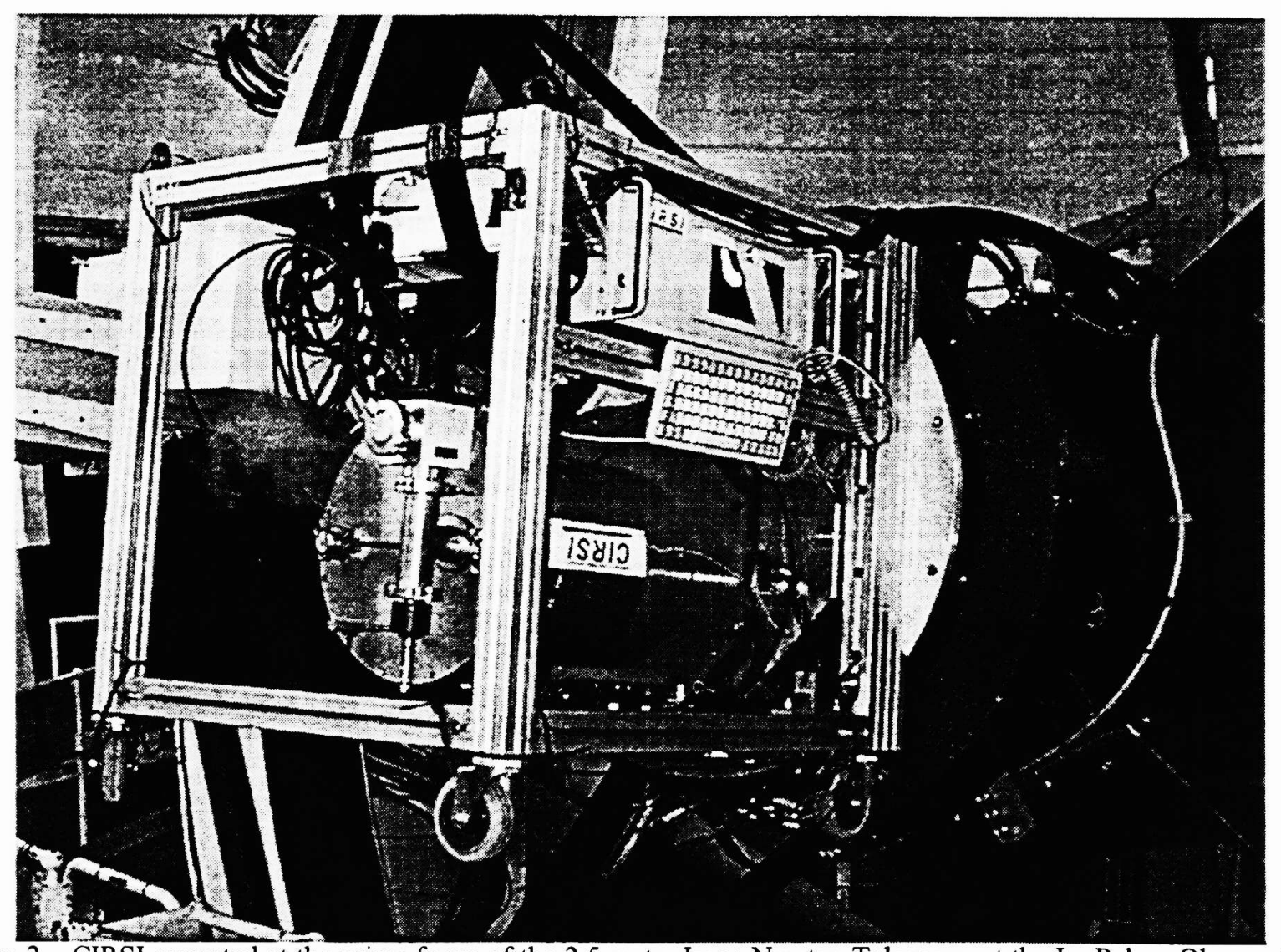

Figure 2. CIRSI mounted at the prime focus of the 2.5 metre Isaac Newton Telescope at the La Palma Observatory, Canary Islands. 


\section{CONTROL SOFTWARE ORGANISATION}

In order to minimise development effort we decided to use a commercially available software package that is supplied with the Capella 4100 controller by PerkinElmer Life Sciences. This package is called PixCel and runs under Windows $95 / 98$ on standard commercial PC systems. We were are able to get access to the source code of this package so that it could modified to provide the package with a wide variety of functions specific to infrared detector operation and to the four detector format of CIRSI. The computer system that runs the camera is mounted beside the detector cryostat and travels with it on the telescope (see Fig 2). Direct control of this computer is provided by the package called pcAnywhere from Symantec. This package allows a window on a remote machine (for example in the telescope control room) to display the screen that would be visible on a monitor attached to the originating computer (in this case the one on the telescope next to the camera), and allows the mouse and keyboard operations on the remote computer to be passed directly to the originating machine. With fast network connections (we use 100-base fibre-optic ethernet) good performance is obtained. The computer that drives the camera takes data from the controller into its memory and then saves it remotely over the high speed ethernet link onto a separate computer running Linux. Linux was chosen as the operating system for the data storage computer because it allows efficient interleaving of network data transfers, data storage and data backup.

The modifications added to the PixCel package allow, amongst other things, the ability to read out individual quadrants or detectors in whatever combination is required, optional automatic reset subtraction, optional assembly of quadrants into full 1024 by 1024 images and the ability to save FITS format data files with headers that include a substantial amount of telescope information derived from the local telescope control system.

Although the pcAnywhere package allows direct control of the camera control computer, something that is essential for setup and diagnostic work, in practice it is much more convenient if the entire system can be run remotely from a script executing on the telescope control computer system. The PixCel package has been modified to allow TCP/IP socket commands to be used to control virtually every function of PixCel, and for PixCel to pass status information back to the control script. The control script may also run the telescope itself as well as ensuring that all telescope status information is available to the PixCel package so that the FITS headers can reflect accurately the state of the telescope system at the moment that the data are taken. This system is therefore capable of unattended operation for periods of several hours, limited only by the need for the astronomer to make him on herself feel in some way useful.

\section{OBSERVING STRATEGIES}

When working in the infrared it is important always to remember that the detectors are less uniform than visible CCDs and the sky is very much brighter in the infrared bands because of considerable amounts of $\mathrm{OH}$ emission from the upper atmosphere. Because of this a major part of the observing strategy is always to take data that allows a clear distinction between those features of the image which are due to detector and instrument non-uniformities and which parts of the image genuinely represent objects in the sky. Infrared detectors such as the ones used here suffer from significant numbers of hot pixels (pixels with much higher than average dark current), dead or low sensitivity pixels as well as the usual problems with cosmic ray events being detected in the device. Not only is the night sky very bright but the strength of the emission lines changes on relatively short time scales. These all contribute to spatial non-uniformities in the light distribution of the sky so the actual sky brightness distribution will change significantly on relatively short time scales. Because of this it is important that data are taken in the way that allows them to be fully calibrated by being internally consistent.

This consistency is achieved by ensuring that any observation consists of a sequence of observations each taken with the telescope pointing at positions differing by typically 5-20 arc seconds. Features that stay on the same position in each image are therefore detector/imaging system specific and features that move are therefore real objects in the sky. We normally use a 9 position dither pattern. We then step the telescope in the sequence of four pointings to give a fully filled mosaic. This allows us to cover an area of 30 by 30 arc minutes on the Isaac Newton Telescope, and 13 by 13 arc minutes on the Du Pont 100 inch Telescope. We take regular groups of calibration images which include flat field exposures (where the telescope points at the inside of the film dome which is illuminated with a relatively uniform tungsten lamp) and similar exposures with a lamp turned off (the dome dark exposures). 


\section{THE DATA PROCESSING PIPELINE}

The images shown in Figures 3, 4 and 5 were produced using the IRDR (Infra-Red Data Reduction) software package, an ANSI C software library that we developed to provide a set of reusable IR data processing tools. The IRDR library routines are called from a collection of stand-alone $C$ programs, with CIRSI-specific perl scripts to glue the tasks into an automated pipeline for reducing CIRSI data. The analysis is done in two passes (using object masks created in the first pass to improve sky frame creation in the second pass), similar to packages such as PHIIRS (Hall, Green, and Cohen 1998) and DIMSUM (Stanford, Eisenhardt, and Dickinson 1995). The basic processing steps are: flat field correction, running sky frame subtraction, dither offsets determination, dither set co-addition, and mosaic image creation.

\section{Flatfield correction}

A flat field image per chip is produced by subtracting the stack median of dome flat observations taken with the dome lamp off from the stack median of dome flat observations taken with the lamp on. The flat field images are divided by the mode of the chip 1 flat field to produce a gain map per chip. Bad pixels are identified as outliers in the gain maps (greater than 5 standard deviations away from the median in 15x15-pixel blocks) or pixels with extremely low or high sensitivity ( $>30 \%$ from the median gain). The data frames are multiplied by the inverse of the gain maps.

\section{Sky subtraction}

For each data frame, a sky image is constructed from the robust mean of the eight nearest frames in the observation (dither) sequence and subtracted. Objects detected in the co-added dither sets from the first pass reduction are masked out during sky frame creation in the second pass. The object masks are produced using the CHECKIMAGE_TYPE OBJECTS option to SExtractor (Bertin and Arnouts 1996) ${ }^{2}$, which sets non-object pixels to 0. The object regions (detection isophotes) generated by SExtractor are "grown" (scaled) by a multiplicative factor of 1.5, and not used in calculating the sky image.

\section{Offset determination}

A typical dither sequence consists of nine observations in a $3 \times 3$ grid with offsets in each direction of approximately 10 arcsec. The approximate dither offsets stored in the FITS header WCS information are refined using cross-correlation analysis. The object pixels of each dither frame (obtained using SExtractor as described above) are cross-correlated against the object pixels of the first dither frame in the sequence. A parabola is fit to the peak of the cross-correlation image to measure the dither offsets relative to the first frame with a precision of about 0.2 pixels.

\section{Dither set co-addition}

A weight map is created for each data frame with the weight for pixel i given by: $w_{i}=g_{i} \bullet c t / V$, where $g_{i}$ is the gain for pixel $i$ (set to 0.0 for bad pixels), $t$ is the exposure time, and $V$ is the image variance. The data frames and corresponding weight maps in the dither set are registered using bi-linear interpolation. Each output (interpolated) pixel value is calculated from the weighted average of the four closest pixels in the input image (discarding bad pixels), with weights given by the fractional areas of overlap. The weight maps are registered similarly but using a weighted sum instead of weighted average.

The dither frames are combined by calculating the weighted mean pixel value at each $(x, y)$ position of the dither stack, with pixel values greater than 5 sigma from the median at each position rejected. Images borders are added during registration to avoid clipping the data to the intersection of the dither frames. The standard deviation (sigma) at each position is calculated from the median absolute deviation if there are at least five valid pixels, otherwise the assumption of photon noise is used. The weight maps are combined by calculating the sum at each $(\mathrm{x}, \mathrm{y})$ position of the stack of weight maps.

\section{Mosaic creation}

SExtractor object detections in the combined dither sets are matched to the APM catalog (McMahon and Irwin 1992, http://www.ast.cam.ac.uk/apmcat $)^{5}$ using a triangle matching program (Valdes et al. 1995) ${ }^{7}$, and the FITS WCS header information is updated. The combined dither sets and weight maps from different chips, telescope pointings, and nights 
are drizzled onto mosaic images using Drizzle (Hook and Fruchter1997, http://www.eso.org/eis $)^{3}$. A mosaic image and associated weight map for a low Galactic latitude field are shown in Figures 3 and 4. A zoomed image of the central region of that field is shown in Figure 5.
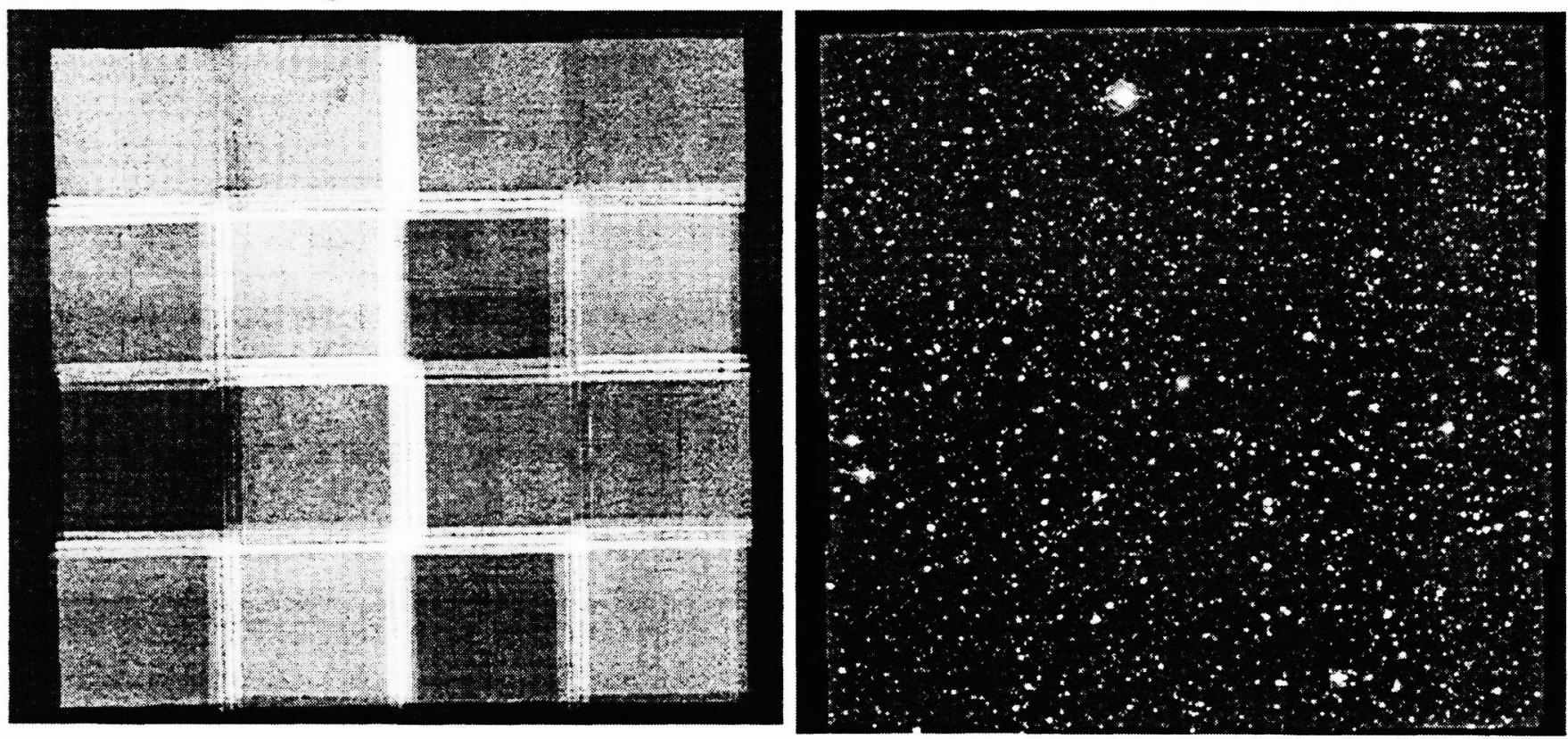

Figures 3 (Left) and 4 (Right): A composite mosaic of four pointings each of which is derived from a nine-point dither. The weighting of the various pixels (essentially the relative exposure time per pixel) is on the left and the data after passing through the data processing pipeline is shown on the right.

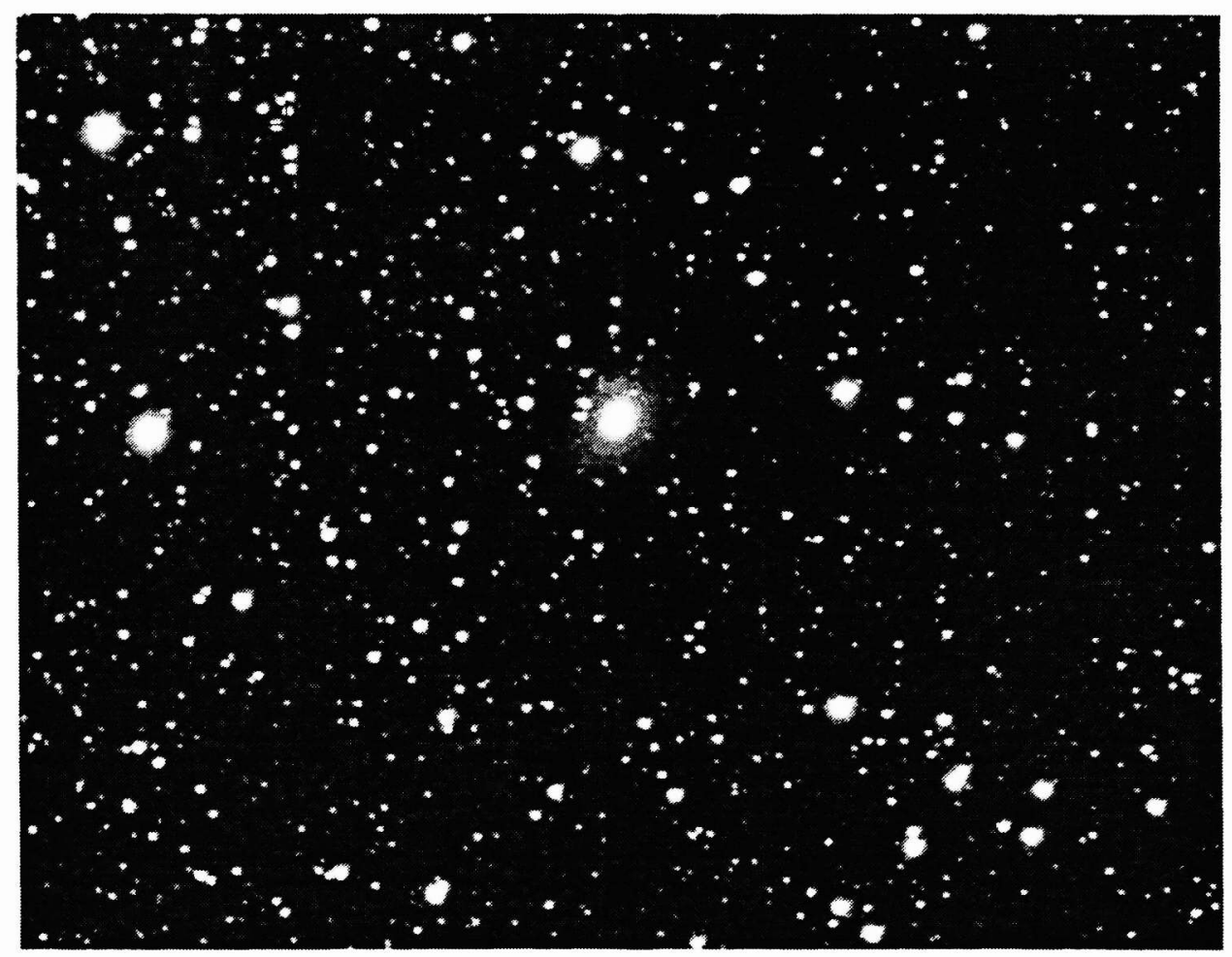

Figure 5: The central region of the field shown in figures 3 and 4. The central galaxy was located in the middle of the array. Despite the conciderable variations in local weights in that area, the image shows no artefacts (other than caused by the printer) and is smooth and continuous across this region. 


\section{SCIENTIFIC PROGRAMMES AND RESULTS}

CIRSI is being used for a number of key scientific programs. At the prime focus of the $2.5 \mathrm{~m}$ Isaac Newton Telescope on La Palma, the relatively coarse scale is $0.45^{\prime \prime} /$ pixel. Thus 4 exposures with CIRSI delivers a contiguous field of $29^{\prime} \times 29^{\prime}$. This makes it ideally suited to large area programs with limiting magnitudes of $\mathrm{H}=19-20$. Example programs are searches for $\mathrm{z}=1$ clusters of galaxies, surveys for brown dwarfs and the identification of serendipitous X-ray sources detected by XMMNewton and Chandra.

At the prime focus of the larger aperture $4.2 \mathrm{~m}$ William Herschel Telescope, CIRSI has a pixel size of 0.32 " and thus is better suited for program where depth and image quality are a requirement. Here CIRSI has been used for gravitational lensing studies of clusters of galaxies. For example, Gray, Ellis etal (Cambridge, UK) measured the gravitational depletion signal at near-infrared wavelengths in the cluster Abell $2219(\mathrm{z}=0.22)$ In this experiment the cluster was centred on a single chip and two adjacent CIRSI images were used to show that the galaxy surface density was depleted at the 3 sigma confidence level due to gravitational lensing effects.

The unique wide field and portability of CIRSI has meant that we have not wanted to be restricted to just Northern Hemisphere targets. Therefore for 6 months of each year CIRSI is a visitor instrument at the $2.5 \mathrm{~m}$ OCIW Du Pont Telescope at Las Campanas, Chile. Here the delivered pixel at the $\mathrm{f} / 7.5$ cassegrian focus is $0.20 "$. The smaller field of view that the INT is partially compensated for by the better seeing and photometric conditions.

Example programs here have been, deep stellar population studies of Cen-A (Ferguson, Cambridge, UK)), a deep confusion limited survey of the galactic centre and inner bulge region (Gilmore, Cambridge, UK) and a deep multi-wavelength photometric redshift survey (McMahon etal, Cambridge, UK). The photometric (UBVRIZ-JHK) redshift survey is being carried out with colleagues at OCIW (McCarthy etal, OCIW, Pasadena, US) and covers a total area of $1 \mathrm{deg}^{2}$ to $\mathrm{K}=21.0$; $\mathrm{H}=21.5 ; \mathrm{J}=22.5 ; \mathrm{V}=27.0$ i.e. a unevolved elliptical galaxy is detectable out to $\mathrm{z} \sim 2$. The primary goal of this program is to trace the evolution of galaxies and their clustering properties on $\mathrm{Mpc}$ scales out to $\mathrm{z}=2$.

\section{CONCLUSIONS}

CIRSI is the first infrared imager to use an array of detectors. It has now been on a telescope for over 100 nights and taken in excess of 0.5 Terabytes of data. The quality of the images is excellent and now that we have an effective data processing pipeline, the data produced is breaking new ground in our understanding of the most distant clusters of galaxies Planned upgrades to CIRSI include a new parallel read-out architecture and faster networking and data archiving, both of which significantly limit our overall data taking efficiency at present. . Larger arrays will become possible in a few years with the advent of larger (2048x2048) detectors, but in the mean time, CIRSI has a great deal of work to do.

\section{ACKNOWLEDGEMENTS}

None of this work would have been possible without the interest and very generous donation of the Raymond and Beverly Sackler and the Trustees of their Foundation. We are also grateful for the help provided by Kadri Vural and his team at the Rockwell Science Centre in California. The telescope staffs of the Isaac Newton Group of telescopes on La Palma, Canary Islands and at the Observatory at Las Campanas in Chile have also provided considerable amount of help.

\section{REFERENCES}

1. M.G. Beckett, C.D. Mackay, R.G. McMahon, I.R. Parry, R.S. Ellis, S.J. Chan, M. Hoenig (1998) CIRSI: Progress with the Cambridge Infra Red Survey Instrument (SPIE, vol. 3354, p431-435, 1998)

2. Bertin, E., and Arnouts, S. 1996, A\&AS, 117, 393

3. Hook, R.N., and Fruchter, A.S. 1997, in ADASS VI, ASP Conf. Ser., Vol. 125, eds. Gareth Hunt and H.E. Payne, 147

4. Hall, P.B., Green, R.F., and Cohen, M. 1998, ApJSS, 119, 1

5. McMahon, R.G. and Irwin, M.J., 1992, in Digitised Optical Sky Surveys, eds. H.T. MacGillivray and E.B.Thomson, Astrophysics and Space Science Library, 174, 417

6. Stanford, S.A., Eisenhardt, P.R.M., and Dickinson, M. 1995, ApJ, 450, 512

7. Valdes, F.G., Campusano, L.E., Velasquez, J.D., and Stetson, P.B. 1995, PASP, 107, 1119 